\title{
Positive geometries for all scalar theories from twisted intersection theory
}

\author{
Nikhil Kalyanapuram ${ }^{1,2, *}$ and Raghav G. Jha $\odot^{1, \dagger}$ \\ ${ }^{1}$ Perimeter Institute for Theoretical Physics, 31 Caroline Street North, Waterloo, Ontario, Canada N2L $2 Y 5$ \\ ${ }^{2}$ Department of Physics and Astronomy, University of Waterloo, Waterloo, Ontario, Canada N2L 3G1
}

(Received 20 April 2020; accepted 26 June 2020; published 22 July 2020)

\begin{abstract}
We show that accordiohedra furnish polytopes which encode amplitudes for all massive scalar field theories with generic interactions. This is done by deriving integral formulas for the Feynman diagrams at the tree level and integrands at the one-loop level in the planar limit using the twisted intersection theory of convex realizations of the accordiohedron polytopes.
\end{abstract}

DOI: 10.1103/PhysRevResearch.2.033119

\section{INTRODUCTION}

The study of scattering amplitudes in the past decade has revealed a number of surprising connections with mathematics. Crucially, deep ties to geometry, topology, and combinatorics [1-36] have been established, which have led to the discovery of new ways of computing these amplitudes.

In this work we focus on building upon the seminal developments in the past few years, namely, the positive geometry program due to Arkani-Hamed et al. [7] and the twisted intersection theory of Mizera [2]. In these works it was seen that for a wide class of theories built out of trivalent vertices, the planar Feynman diagrams are encoded by the geometry of a polytope known as the associahedron. This was extended to massless scalar theories with generic interactions in [17], in which a polytope known as the accordiohedron was introduced. In this paper we propose a broad generalization of this line of research by applying the technology of intersection theory to the accordiohedron polytopes.

We seek to address two open questions in the literature. These are as follows. So far, attention has been restricted to the handling of massless interacting particles. The reason for this is the specific realization of the associahedra as convex polytopes, which puts severe restrictions on the masses of the interacting particles. Here we extend the positive geometry program to all scalar theories while utilizing a convex realization of accordiohedra that removes this restriction on the mass and are thus able to treat without any difficulty the interactions between particles of arbitrary mass.

As far as the positive geometry program is concerned, loop effects have been difficult to incorporate. Technical restrictions have forced us to only deal with $\phi^{3}$ interactions among massless particles at the one-loop level. We rectify

\footnotetext{
*nkalyanapuram@perimeterinstitute.ca

${ }_{\dagger}^{\dagger}$ rjha1@ perimeterinstitute.ca
}

Published by the American Physical Society under the terms of the Creative Commons Attribution 4.0 International license. Further distribution of this work must maintain attribution to the author(s) and the published article's title, journal citation, and DOI. this by proposing a class of accordiohedra which describes interactions between particles in any scalar theory at the oneloop level, in the planar limit. Our construction also allows us to handle different kinds of Feynman diagrams separately, for example, allowing us to treat tadpoles and bubble diagrams distinctly.

Let us briefly discuss what has is done in the paper and the organization of the text. What is accomplished is a generalization of the positive geometry framework to take care of massive particles as well. This is done in Sec. II. Following this, in Sec. III we also describe a simple example indicating that the story can be pushed to at least one-loop order in arbitrary theories and point out the problems involved in higher-loop cases. In doing so we rectify a problem which has been ignored in the literature, namely, the handling of symmetry factors in Feynman diagrams.

\section{MASSIVE $\phi^{3}+\phi^{4}$ SCALAR THEORIES}

In this section we describe how the twisted intersection theory of accordiohedra can be used to compute scattering amplitudes for generic scalar theories involving massive particles.

Much of the work on positive geometries for scalar theories beyond $\phi^{3}$ has been done quite recently. For the case of $\phi^{4}$ and $\phi^{p}$ interactions, the relevant papers are [12,16], respectively. The formalism for studying generic theories was worked out in [17]. Conspicuously, the analysis in these papers worked specifically for massless particles.

In this section we illustrate how the positive geometry formalism can accommodate massive particles through a development of the intersection theory governing amplitudes in massive scalar theories with $\phi^{3}+\phi^{4}$ interactions. It will turn out that this is the right arena to generalize the study of polytopes controlling these amplitudes for massless particles to massive ones. To do this, we make use of the accordiohedron data first presented in [17] and the method of realizing these as convex polytopes reviewed in [37]. To keep the discussion simple, let us restrict ourselves to the case of six-particle scattering. This particular process gives rise to two classes of accordiohedra, namely, squares and pentagons. Let us begin with the square, which is obtained from the 


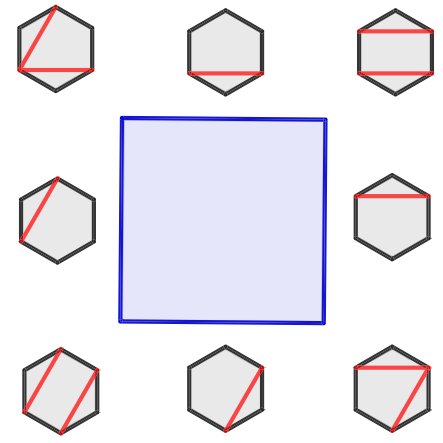

FIG. 1. Two-dimensional accordiohedron for the reference dissection $(13,46)$. The reference dissection is at the top right.

dissection $(13,46)$. The accordiohedron vertices are labeled by $\{(13,46),(24,46),(26,35),(13,35)\} .^{1}$ Accordingly, the codimension-1 boundaries are labeled by the partial dissections $\{(13),(46),(26),(35)\}$. This is illustrated by Fig. 1.

The next task is to find a suitable convex embedding of this polytope as a hyperplane arrangement in $\mathbb{C} \mathbb{P}^{2}$, which is rendered possible due to the generic form of the polytopal realization reviewed in [37]. The hyperplanes for an accordiohedron are obtained by comparing the diagonals labeling the facets with the reference dissection. Starting with the facet (13), we have to compare it to the reference dissection (see Fig. 2).

We see from Fig. 2 that the dissection (13) intersects the reference (13) and forms an inverted Z (see Fig. 1 of [38]) and does not intersect (46) at all. Using the rules reviewed in [37], we can write the facet (13) (denoted by $f_{1}$ ) as

$$
\left(x \hat{e}_{13}+y \hat{e}_{46}\right) \cdot\left(\hat{e}_{13}+0 \hat{e}_{46}\right) \leqslant 1 \Rightarrow x \leqslant 1 .
$$

Here we have used a basis for $\mathbb{C P}^{2}$ with basis vectors $\hat{e}_{13}$ and $\hat{e}_{46}$. In addition, $x$ and $y$ are the respective values of the inhomogeneous coordinates. Using the same rules, we can now write the facets (46), (35), and (26) (denoted by $f_{2}, f_{3}$, and $f_{4}$ ) as

$$
y \leqslant 1, \quad y \geqslant-1, \quad x \geqslant-1 .
$$

Clearly, these hyperplanes bound a square. Now we can shift our interest to the configuration space which is the reference manifold with four hyperplanes above and at infinity removed, i.e., $\mathbf{X}=\mathbb{C P}^{2}-\bigcup_{i=1}^{4} f_{i}{ }^{2}$ On this space $\mathrm{X}$, we define the twist

$$
\begin{aligned}
\omega_{(13,46)}= & \left(X_{13}-m_{13}^{2}\right) d \ln (x-1)+\left(X_{26}-m_{26}^{2}\right) d \ln (x+1) \\
& +\left(X_{46}-m_{46}^{2}\right) d \ln (y-1) \\
& +\left(X_{35}-m_{35}^{2}\right) d \ln (y+1)
\end{aligned}
$$

\footnotetext{
${ }^{1}$ We use the notation for dissections set up in [12]. For example, $(13,46)$ means that a hexagon is dissected by drawing a diagonal between vertices 1 and 3 and a diagonal between 4 and 6 .

${ }^{2}$ We have not explicitly indicated the hyperplane at infinity, which is formally present. The residue at infinity can be computed by a simple change of variables. It does not however affect our computation of the intersection number.
}

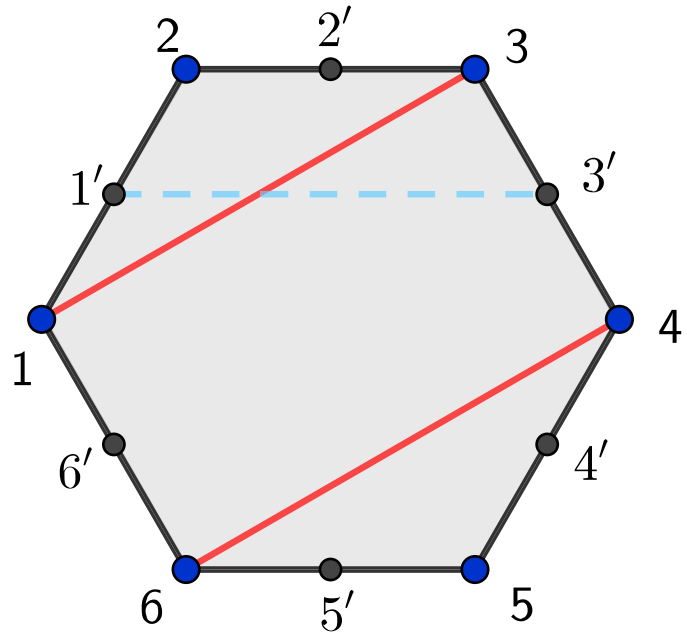

FIG. 2. Comparison of the dissection (13) (denoted by the dashed line) with the reference $(13,46)$ (denoted by red solid lines).

We have used the standard notation to describe generalized Mandelstam variables, i.e., $X_{i j}$ is equal to $\left(p_{i}+p_{i+1}+\cdots+\right.$ $\left.p_{j-1}\right)^{2}$. These can be visualized as chords of an $n$-gon for an $n$-particle scattering process. Consequently, the dissection $(13,46)$ will translate into the diagram having poles as $X_{13}$ and $X_{46}$ go on-shell. It can be seen from this picture that there are $\frac{n(n-1)}{2}-n$ such variables, which is precisely the dimensionality of the space of Mandelstam variables for an $n$-particle process.

Let us also note the meaning of the notation $m_{i j}^{2}$. For purposes of maximal generality, we assume that each channel of the scattering process has a different massive pole. Here $m_{i j}^{2}$ is the squared mass of the particle propagating along the channel $(i j)$. Now, for the case of a theory with a single kind of particle, all the $m_{i}^{2} j$ will be equal to $m^{2}$, where $m^{2}$ is the mass of the particle. Since we can work out the intersection theory for arbitrary masses, we note that this formalism can be applied for amplitudes such as those in thermal field theories as well, where the $m_{i j}^{2}$ can be identified with Matsubara frequencies. Thus, we can deal with a fairly wide class of theories using this framework.

With this laid out, we can compute the contribution to the scattering amplitude from this polytope by computing the selfintersection number of the form

$$
\begin{aligned}
\varphi_{(13,46)}= & d \ln f_{1} \wedge d \ln f_{2}+d \ln f_{2} \wedge d \ln f_{3} \\
& +d \ln f_{3} \wedge d \ln f_{4}+d \ln f_{4} \wedge d \ln f_{1} .
\end{aligned}
$$

This can be seen from the formula for intersection numbers, which was first used in the context of scattering amplitudes in [2]. In our case, we are interested in the self-intersection number of $\varphi_{(13,46)}$, for which it is sufficient to note that the intersection number is localized on the vertices of the accordiohedron. Schematically, for a given accordiohedron of dimension $n$, if the vertices are labeled by $V_{I}$, the selfintersection number of the corresponding form would be given by

$$
\sum_{f_{1} \cap \cdots \cap f_{n}=V_{I}} \frac{1}{\alpha_{1} \cdots \alpha_{n}},
$$




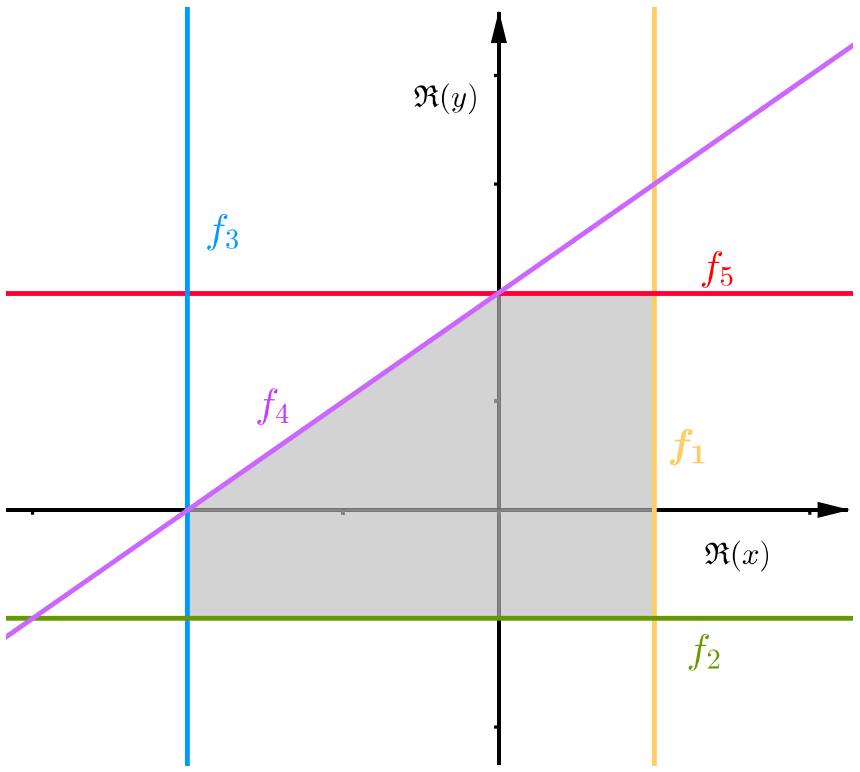

FIG. 3. Accordiohedron for the dissection $(13,14)$ embedded in $\mathbb{C} \mathbb{P}^{2}$.

where $\alpha_{i}$ is the weight attached to $f_{i}$. In our case, an application of this formula to $\varphi_{(13,46)}$ gives

$$
\begin{aligned}
\left\langle\varphi_{(13,46)}, \varphi_{(13,46)}\right\rangle & \frac{1}{X_{13}-m_{13}^{2}} \frac{1}{X_{46}-m_{46}^{2}}+\frac{1}{X_{46}-m_{46}^{2}} \frac{1}{X_{35}-m_{35}^{2}} \\
& \times \frac{1}{X_{35}-m_{35}^{2}} \frac{1}{X_{26}-m_{26}^{2}}+\frac{1}{X_{26}-m_{26}^{2}} \frac{1}{X_{13}-m_{13}^{2}} .
\end{aligned}
$$

A similar approach can be taken for the pentagon arising from the six-particle amplitude in this theory shown in Fig. 3. We will focus on the reference dissection $(13,14)$, which gives rise to a pentagon. The accordiohedron of this reference is labeled by the vertices $\{(13,14),(24,14),(24,26),(26,36),(13,36)\}$. The facets may be read off from the set of vertices; they are $\{(13),(36),(26),(24),(14)\}$. Using the rules for finding the embedding, we have the facets (denoted by $f_{1} \cdots f_{5}$ )

$$
x \leqslant 1, \quad-y \leqslant 1, \quad-x \leqslant 2, \quad-x+y \leqslant 2, \quad y \leqslant 2 .
$$

These constraints give rise to the shaded convex polygon in Fig. 3. The kinematical data associated with the amplitude is carried by the twist, which we choose as

$$
\begin{aligned}
\omega_{(13,14)}= & \left(X_{13}-m_{13}^{2}\right) d \ln (x-1)+\left(X_{14}-m_{14}^{2}\right) d \ln (y-2) \\
& +\left(X_{24}-m_{24}^{2}\right) d \ln (-x+y-2) \\
& +\left(X_{26}-m_{26}^{2}\right) d \ln (x+2) \\
& +\left(X_{36}-m_{36}^{2}\right) d \ln (y+1)
\end{aligned}
$$

Using the assignments for the $f_{i}$ as defined by Fig. 3, we compute the self-intersection number of the form

$$
\begin{aligned}
\varphi_{(13,14)}= & d \ln f_{1} \wedge d \ln f_{2}+d \ln f_{2} \wedge d \ln f_{3} \\
& +d \ln f_{3} \wedge d \ln f_{4}+d \ln f_{4} \wedge d \ln f_{5} \\
& +d \ln f_{5} \wedge d \ln f_{1}
\end{aligned}
$$

to compute the amplitude, which becomes

$$
\begin{aligned}
& \left\langle\varphi_{(13,14)}, \varphi_{(13,14)}\right\rangle \\
& =\frac{1}{X_{13}-m_{13}^{2}} \frac{1}{X_{36}-m_{36}^{2}}+\frac{1}{X_{36}-m_{36}^{2}} \frac{1}{X_{26}-m_{26}^{2}} \\
& \quad+\frac{1}{X_{26}-m_{26}^{2}} \frac{1}{X_{24}-m_{24}^{2}}+\frac{1}{X_{24}-m_{24}^{2}} \frac{1}{X_{14}-m_{14}^{2}} \\
& \quad+\frac{1}{X_{14}-m_{14}^{2}} \frac{1}{X_{13}-m_{13}^{2}} .
\end{aligned}
$$

We have indicated that the twist and form are defined for the particular accordiohedron in question by using the subscript $(13,46)$ to denote the reference dissection.

These calculations show that arbitrary mass choices can be made perfectly consistent in the polytopes formalism, even though this aspect is not manifest in the conventional embedding in the kinematical space. It is then obvious that the natural arena for massive scalar theories is twisted intersection theory with a careful convex realization of accordiohedra, which allows us to study scalar theories with arbitrary masses.

We note here that for theories in which a number of massive states can be exchanged in the Mandelstam channels, the amplitude will be given over a sum of intersection numbers; no single intersection number can give all the amplitudes summed over. The two index masses simply provide a general scheme to consider any massive pole structure.

\section{INCORPORATING LOOP EFFECTS}

A proper discussion including loops while considering scattering amplitudes from the positive geometry viewpoint has been met with some hurdles. For one thing, it has been difficult to make swift progress beyond one-loop Feynman diagrams, due to the technical difficulties in dealing with moduli spaces of genus 2 surfaces. To be precise, these surfaces are not known to be tiled by any regular polytope, making the analysis somewhat tricky. Some progress has been reported at genus 1 , for which the reader can consult $[13,14,39]$.

In addition to the general technical issue of looking at moduli spaces, there is a more mundane issue with including loop interactions. Generically, the integrands appearing in Feynman loop diagrams come with symmetry factors, which encode various degeneracies arising from the large number of ways in which contractions can be performed.

For these reasons, it may be more efficient to look at specific classes of Feynman diagrams depending on the nature of renormalization and see if these classes can be described in the polytope framework. To be more concrete, let us consider the case of four-particle scattering (in the planar limit) in $\phi^{4}$ theory. Here we receive contributions from two classes of diagrams, namely, from diagrams which cause mass renormalization and from diagrams giving rise to coupling constant renormalization.

In order to recast these as intersection numbers, we follow the algorithm that we will now describe. In the field theory limit, which is what we are interested in for the time being, loop interactions are encoded by the complete nodal degeneration of the moduli space $\mathcal{M}_{g, n}$, which is $\mathcal{M}_{0, n+2 g}$. Given the $2 g$ auxiliary insertions, denoted by $\sigma_{ \pm, i}$, with $i$ running from 

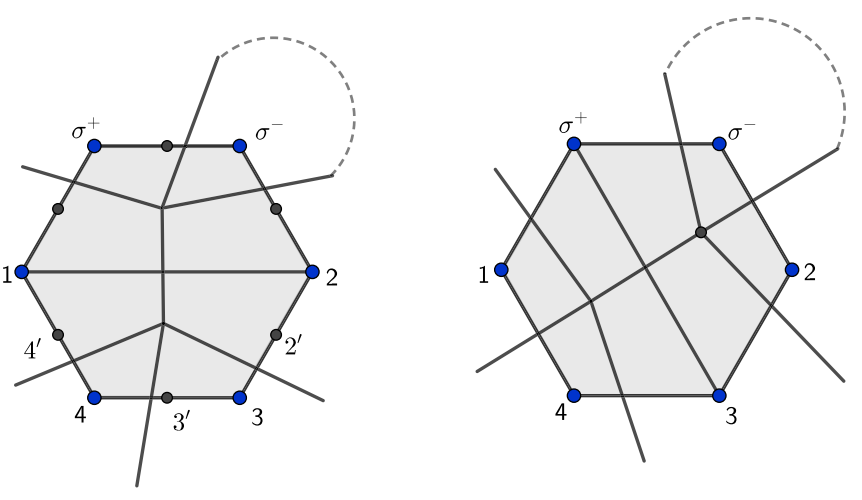

FIG. 4. Propagator corrections in the $\phi^{4}$ theory at the one-loop level.

1 through $g$, each pair can be sandwiched between a pair of the original insertions as shown in Fig. 4. All possible ways of doing this constitute the tiling of the moduli space.

Let us specialize to the case of the four-particle scattering described earlier. Specifically, let the auxiliary points be placed between particles 1 and 2. Furthermore, these two insertions are associated with momenta $\ell$ and $-\ell$. If we now look at only the terms giving rise to mass renormalization, we have two diagrams as shown in Fig. 4. These two diagrams are obtained from the dissections $(12)$ and $(+3)$. Here $(+3)$ indicates a diagonal between the vertex $\sigma^{+}$and 3. Using these dissections, the technology of accordiohedra and intersection theory may be applied to obtain the stripped integrand, namely, the integrand with the loop momentum stripped.

We first find the accordiohedra for the two dissections. For (12), the only compatible dissection is itself. This gives an open accordiohedron, in which the second boundary is pushed to infinity. However, for $(+3)$, the accordiohedron is $\{(12),(+3)\}$. Thus, the weights are 0 and 1 , respectively. We can realize this as $\mathbb{C} \mathbb{P}^{1}-\{0,1, \infty\}$ with the twist

$$
\begin{aligned}
\omega_{(+3)}= & \left(p_{1}^{2}-m^{2}\right) d \ln (x) \\
& +\left(p_{2}^{2}-\mu^{2}\right) d \ln (x-1),
\end{aligned}
$$

where $m$ and $\mu$ are the masses of virtual particles flowing through the respective channels and the hyperplane at infinity has been indicated. Now the self-intersection number of $\varphi_{(+3)}=d \ln \left(\frac{x}{x-1}\right)$ gives

$$
\frac{1}{p_{1}^{2}-m^{2}}+\frac{1}{p_{2}^{2}-\mu^{2}} .
$$

If the loop momentum is introduced, we get

$$
\frac{1}{\ell^{2}}\left(\frac{1}{p_{1}^{2}-m^{2}}+\frac{1}{p_{2}^{2}-\mu^{2}}\right),
$$

which is the correct loop integrand. Indeed, this can be absorbed as a renormalization of mass after all channels are taken into account. For this of course we have to analytically continue past the mass shell, which the intersection theory does not preclude.

Extending this to loop levels higher than one has a technical issue, namely, the fact that stripping away all the loop momenta as $\prod_{i} \frac{1}{\ell_{i}^{2}}$ is not generically possible, due in large part to the fact that Riemann surfaces of genus greater than 2 can degenerate in very complicated ways to give rise to nodal Riemann spheres. The extension of the results obtained here to higher loop order remains an interesting open problem.

\section{GENERIC INTERACTIONS}

In this section we briefly describe how the procedure developed above may be applied to generic theories. Let us first note that the main object of importance is the so-called accordiohedron, constructed out of a given set of dissections, which label a particular scattering process. Most importantly, these scattering processes can be arbitrarily complicated, so long as the dissections are properly classified and treated appropriately.

Consider, for example, the rather complicated kinds of polytopes considered in [16], in which the accordiohedra for arbitrary $\phi^{p}$ interactions were obtained. Here dissections of $p+n(p-2)$-gons into $p$-gons label the collection of all planar Feynman diagrams in an $n$-particle scattering process. Accordingly, the collection of these dissections may be used to obtain the corresponding accordiohedra, which may then be realized as convex polytopes using the methods used here, which were reviewed in [37].

At the same time, we must also bear in mind that there is a practical hurdle to all of this. Leaving aside the computationally intensive aspect, we also recall that accordiohedra are not generically unique, and a number of distinct accordiohedra usually need to be appropriately weighted and resummed in order to obtain the final amplitude. In our case, this will entail appropriately weighting the corresponding twisted intersection numbers.

From this discussion, the takeaway is simply that the formalism itself can be applied rather straightforwardly, even if cumbersome, such that the real roadblock is to ensure that a self-consistent collection of weights can be obtained. Indeed, determining whether or not these weights can be found consistently was an important aspect considered during the work that led to [17], with some very decent progress also discussed in [16]. In all the cases considered so far, the weights can be determined consistently. Furthermore, in [17] it was found that there are at most as many equations determining the weights as there are weights, consequently implying that at least one self-consistent solution may be found.

To conclude this section, we remark that the previous points indicate that the procedure outlined in this paper can be carried out for arbitrarily complicated interactions, which, although technically challenging at higher points, will always be possible in principle.

\section{DISCUSSION}

In this paper we have developed a framework to handle interactions among scalars in the planar limit which may be arbitrarily complicated from the point of view of twisted intersection theory. Furthermore, we have noted that the formalism presented circumvents some of the rules that are placed on more traditional amplituhedron methods, chief among which is the restriction to massless particles. The convex embedding 
allows for arbitrary choices of mass as well as moving off the mass shell. Among other things, this allows us to treat tadpoles and bubble diagrams with relative ease. Furthermore, we have been able to bring loop amplitudes, at least up to the one-loop level, into the discussion as well while taking care of symmetry factors.

It seems that there are some aspects of this work which can be easily extended. First, in order to keep track of symmetry factors at the loop level, we have by hand been restricted to specific subsets of dissections giving rise to loop diagrams according to the nature of renormalization (e.g., mass renormalization and coupling constant renormalization in $\phi^{4}$ are treated separately). It remains to be seen whether the symmetry factors and all loop diagrams can be consistently reconciled with one another in the polytopes picture. This seems unlikely, but will surely constitute an interesting future investigation.

Second, it may be interesting to extend our analysis past the realm of scalar theories into richer domains, such as effective field theories (EFTs). Historically, the Cachazo-HeYuan formalism has provided ample insight into EFTs which can be obtained by dimensional reduction of gravity and Yang-Mills theory. Now the technology developed here to understand more generic vertices might give us room to look at more exotic EFTs. This is a long-term goal that we hope to pursue in the future.

\section{ACKNOWLEDGMENTS}

We thank Jacob Bourjaily and Sebastian Mizera for going over the draft and helpful comments. We thank Alfredo Guevara and Seyed Faroogh Moosavian for discussions. We thank an anonymous referee for suggesting clarification. Research at Perimeter Institute was supported in part by the Government of Canada through the Department of Innovation, Science and Economic Development Canada and by the Province of Ontario through the Ministry of Colleges and Universities.
[1] S. Mizera, Combinatorics and topology of Kawai-Lewellen-Tye relations, J. High Energy Phys. 08 (2017) 097.

[2] S. Mizera, Scattering Amplitudes from Intersection Theory, Phys. Rev. Lett. 120, 141602 (2018).

[3] S. Mizera, Aspects of scattering amplitudes and moduli space localization, Ph.D. thesis, Perimeter Institute for Theoretical Physics, 2019.

[4] N. Arkani-Hamed, J. L. Bourjaily, F. Cachazo, A. B. Goncharov, A. Postnikov, and J. Trnka, Grassmannian Geometry of Scattering Amplitudes (Cambridge University Press, Cambridge, 2016).

[5] N. Arkani-Hamed and J. Trnka, The amplituhedron, J. High Energy Phys. 10 (2014) 030.

[6] N. Arkani-Hamed, A. Hodges, and J. Trnka, Positive amplitudes in the amplituhedron, J. High Energy Phys. 08 (2015) 030.

[7] N. Arkani-Hamed, Y. Bai, S. He, and G. Yan, Scattering forms and the positive geometry of kinematics, color and the worldsheet, J. High Energy Phys. 05 (2018) 096.

[8] N. Arkani-Hamed, Y. Bai, and T. Lam, Positive geometries and canonical forms, J. High Energy Phys. 11 (2017) 039.

[9] N. Arkani-Hamed, H. Thomas, and J. Trnka, Unwinding the amplituhedron in binary, J. High Energy Phys. 01 (2018) 016.

[10] N. Arkani-Hamed, S. He, and T. Lam, Stringy canonical forms, arXiv:1912.08707.

[11] N. Arkani-Hamed, T. Lam, and M. Spradlin, Non-perturbative geometries for planar $\mathcal{N}=4$ SYM amplitudes, arXiv:1912.08222.

[12] P. Banerjee, A. Laddha, and P. Raman, Stokes polytopes: The positive geometry for $\phi^{4}$ interactions, J. High Energy Phys. 08 (2019) 067

[13] G. Salvatori and S. L. Cacciatori, Hyperbolic geometry and amplituhedra in 1+2 dimensions, J. High Energy Phys. 08 (2018) 167.

[14] G. Salvatori, 1-loop amplitudes from the halohedron, J. High Energy Phys. 12 (2019) 074.
[15] P. B. Aneesh, P. Banerjee, M. Jagadale, R. R. John, A. Laddha, and S. Mahato, On positive geometries of quartic interactions II: Stokes polytopes, lower forms on associahedra and worldsheet forms, J. High Energy Phys. 04 (2020) 149.

[16] P. Raman, The positive geometry for $\phi^{p}$ interactions, J. High Energy Phys. 10 (2019) 271.

[17] P. B. Aneesh, M. Jagadale, and N. Kalyanapuram, Accordiohedra as positive geometries for generic scalar field theories, Phys. Rev. D 100, 106013 (2019).

[18] E. Witten, Perturbative gauge theory as a string theory in twistor space, Commun. Math. Phys. 252, 189 (2004).

[19] R. Roiban, M. Spradlin, and A. Volovich, On the tree level $S$ matrix of Yang-Mills theory, Phys. Rev. D 70, 026009 (2004).

[20] R. Roiban, M. Spradlin, and A. Volovich, A googly amplitude from the B model in twistor space, J. High Energy Phys. 04 (2004) 012.

[21] F. Cachazo, S. He, and E. Y. Yuan, Scattering equations and Kawai-Lewellen-Tye orthogonality, Phys. Rev. D 90, 065001 (2014).

[22] F. Cachazo, S. He, and E. Y. Yuan, Scattering of Massless Particles in Arbitrary Dimensions, Phys. Rev. Lett. 113, 171601 (2014).

[23] F. Cachazo, S. He, and E. Y. Yuan, Scattering in three dimensions from rational maps, J. High Energy Phys. 10 (2013) 141.

[24] F. Cachazo, S. He, and E. Y. Yuan, Scattering of massless particles: Scalars, gluons and gravitons, J. High Energy Phys. 07 (2014) 033.

[25] F. Cachazo, S. He, and E. Y. Yuan, Einstein-Yang-Mills scattering amplitudes from scattering equations, J. High Energy Phys. 01 (2015) 121.

[26] F. Cachazo, S. He, and E. Y. Yuan, Scattering equations and matrices: From Einstein to Yang-Mills, DBI and NLSM, J. High Energy Phys. 07 (2015) 149. 
[27] F. Cachazo, S. He, and E. Y. Yuan, One-loop corrections from higher dimensional tree amplitudes, J. High Energy Phys. 08 (2016) 008

[28] L. Mason and D. Skinner, Ambitwistor strings and the scattering equations, J. High Energy Phys. 07 (2014) 048.

[29] T. Adamo, E. Casali, and D. Skinner, Ambitwistor strings and the scattering equations at one loop, J. High Energy Phys. 04 (2014) 104.

[30] Y. Geyer, L. Mason, R. Monteiro, and P. Tourkine, Loop Integrands for Scattering Amplitudes from the Riemann Sphere, Phys. Rev. Lett. 115, 121603 (2015).

[31] Y. Geyer, L. Mason, R. Monteiro, and P. Tourkine, One-loop amplitudes on the Riemann sphere, J. High Energy Phys. 03 (2016) 114

[32] Y. Geyer, L. Mason, R. Monteiro, and P. Tourkine, Two-loop scattering amplitudes from the Riemann sphere, Phys. Rev. D 94, 125029 (2016).

[33] Y. Geyer and R. Monteiro, Two-loop scattering amplitudes from ambitwistor strings: From genus two to the nodal Riemann sphere, J. High Energy Phys. 11 (2018) 008.

[34] P. Mastrolia and S. Mizera, Feynman integrals and intersection theory, J. High Energy Phys. 02 (2019) 139.

[35] H. Frellesvig, F. Gasparotto, S. Laporta, M. K. Mandal, P. Mastrolia, L. Mattiazzi, and S Mizera, Decomposition of Feynman integrals on the maximal cut by intersection numbers, J. High Energy Phys. 05 (2019) 153.

[36] H. Frellesvig, F. Gasparotto, M. K. Mandal, P. Mastrolia, L. Mattiazzi, and S. Mizera, Vector Space of Feynman Integrals and Multivariate Intersection Numbers, Phys. Rev. Lett. 123, 201602 (2013).

[37] N. Kalyanapuram, Stokes polytopes and intersection theory, Phys. Rev. D 101, 105010 (2020).

[38] T. Manneville and V. Pilaud, Geometric realizations of the accordion complex of a dissection, Discrete Comput. Geom. 61, 507 (2019).

[39] G. Salvatori and S. Stanojevic, Scattering amplitudes and simple canonical forms for simple polytopes, arXiv:1912.06125. 